\title{
Editorials
}

\section{Time to get Mediterranean with our}

\section{dietary advice}

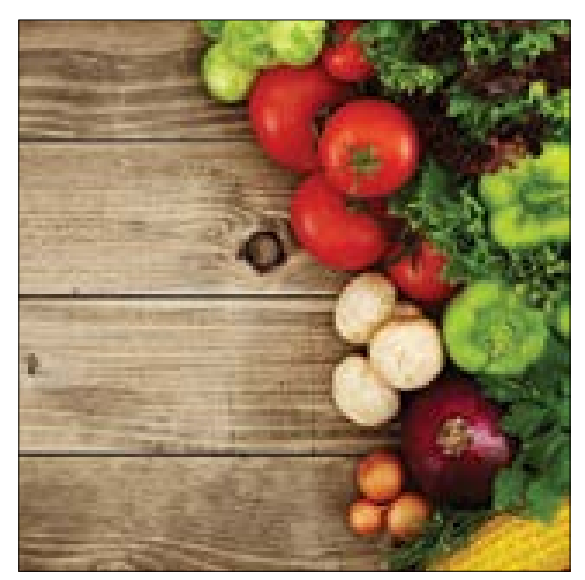

\section{INTRODUCTION}

The Mediterranean diet has long been considered healthy and in particular, was first made by the American doctor Ancel Keys over 50 years ago,' the die has attracted considerable interest in the past decade or so, with a large body potential it has to reduce chronic diseass varying from dementia to cancers ${ }^{23}$ Perhaps the greatest focus, however, remains on the potential it has to reduce morbidity and mortality in cardiovascular diseases (CVD).

THE PREDIMED TRIAL

In April 2013, the results of the long Mediterráneal study were published ${ }^{4}$ This large Spanish trial randomised patients at high risk of CVD to an intervention including advice about the Mediterranean diet alongside supplementation with either olive oils or mixed nuts, or to advise about a lowat diet in the control arm. Interim analyses of results were sufficiently convincing to comparison to the control group, those in the intervention groups had approximately $30 \%$ reductions in their risk of developing CVD.

This level of reduction is remarkable for dietary intervention and will undoubtedy excite many nutritionists and clinicians particulary the difficuly of particularly the difficulty establishing were due to the intensive dietary advice or the supplementation with oil and nuts.
This large Spanish trial randomised patients at high risk of CVD to an intervention including advice about the Mediterranean diet alongside supplementation with either olive oils or mixed nuts.

The pragmatic difficulties of undertaking cinical nutrition trials are well established and indeed, this is the reason why our evidence base in this area. There also main unanswered questions about how tside of the Mediterranean region. where the typical diets may not be entirely amenable to adaptation to features of this diet. As with any landmark study, interpretations of the results have varied extording to the outlook of the reader, with (evels of ontimism and criticism in their light of these compelling results, we may be tempted to start recommending the Mediterranean diet with greater conviction, particularly to those patients who are concerned about, or at high risk of, developing CVD. Anticipating this, some of the important elements of this dietary pattern are rev

THE TRADITIONAL MEDITERRANEAN

The traditional Mediterranean diet existed villages around the Mediterranean Sea fter the Second World War ${ }^{5}$ but global urbanisation and the increased availability of processed goods and fast foods have meant it has become increasingly rare. In a 2010 UNESCO report, the diet was Intangible Cultural Heritage of Humanity

of Spain, Greece, Italy, and Morocco. cor common principles that ru number of The following points summarise some of these common themes.

\section{Fruits and vegetables}

Giving the strong tradition of agriculture 政 may come as little surprise that fresh fruits and vegetables are integral to the diets of point of this dhey are, in fact, the focal point of this dietary pattern, with fruits forming the bulk of both and vegetables evening meals.

\section{Knowing your fats}

an diet is high in monounsaturated and polyunsaturated ats, which increase high-density lipoprotein TDL) levels and are high in antioxidants There is much less room, meanwhile, for . the main source of dietary fot but the mphasis is on using it raw on salads and pread, as heating it may negate some of the positive benefits. Given the high cost of olive oil, recommendations may include cheaper monounsaturated oils such as canola or peanut oil in cooking because bland flavour in the cooked foods.

of the intervention groups in the

light of these compelling results, we may be tempted to start recommending the Mediterranean diet with greater conviction
The health benefits of the Mediterranean diet are clear.

PREDIMED study was assigned to receiving a combination of walnuts, almonds, and hazelnuts with instructions to consume about an ounce of the mix each day. As well as providing unsaturated fats, they form an ideal snack with dried fruits, deterring from unhealthy alternatives such as crisps and

Fish

The benefits of regular fish consumption on both healthy individuals and those with established CVD is well documented and reflected in national CVD guidelines.? With red meat virtually non-existent in the
traditional Mediterranean diet, fish is the ideal replacement. In the PREDIMED trial, consumption at least three times a week was advised in both intervention arms.

Lifestyle

The word 'diet' originates from the Greek word for lifestyle, 'diaita' and the precise contribution of extra-nutritional aspects of the Mediterr anean diet is unclear. As well as rest time, mealtime sociability, modere portion sizes, shared culinary, activities, and seasonality of food choices, all of which are felt to be important contributing factors ${ }^{8}$ Although the PREDIMED trial looked predominantly at older adults at high CVD risk, the diet is sultable for all members of the family. With increasing rates of childhood obesity. communal eating and may be more relevant than ever.

IMPLICATIONS FOR PRIMARY CARE Diets tend to come in and out of fashion and vary in their popularity with patients. The Mediterranean diet, however, is unusual in that it is essentially an inclusion, rather than exclusion diet. The emphasis is on such, although the benefits seen in the PREDIMED study were short and medium term, it essentially represents a long-term dietary change for whole families. It is perhaps better defined as a nutritional model than a diet, reflecting the emphasis on communal meals and intergenerational education about foods. Although the in childhood is less developed, it has been demonstrated that high adherence to can have significant benefits on symptom evidence that CVD is linked to childhood risk factors, ${ }^{10}$ further work examining the value of this diet for children is warranted. GPs have the unique privilege of many generations. They also deal with multimorbidity and health promotion as well as having sufficient continuity in their relationships to allow them to influence lifestyle habits. They are, therefore, in an ideal position to discuss dietary behaviour with patients. The PREDIMED tria demonstrates that the health benefits of economic analyses may be needed before changes at policy level are warranted. There must additionally be a responsibility on clinicians, particularly those in primary care, to begin to distil this information in coherent way to receptive and motivate patients and families.

\section{Mohammed Ahmed Rashid,}

NIHR Academic Clinical Fellow, The Primary Care Unit, Department of Public Health and Primary

\section{Provenance}

DOI: 10.3399/bjgp14X677365
ADDRESS FOR CORRESPONDENCE

\section{Ment}

The Primary Care Unit, Department of Public
Health and Primary Care, University of Cambridge,

Strangeways Research Laboratory, Worts

E-mail: mar74lamedschl.cam.ac.uk

REFERENCES

.
and 15 -year death hate in the seven contries study. Am J Epidemiol 1986; 12466): 903-915. Louridal ISoni M. Thompson-Coon J, et al.
Mediterranean diet, cognitive function, and 2013; 244:4): $779-489$.

LaV Vecchia C. Mediterranean diet and cancer.
Public Health Nutr 2004; 777): $655-968$. Estruch R, Ros E, Salas-Salvadó J, et al. With a Mediterranean diet NEngl/ Medisease

Mrichopoulou A, Lagiou P. Healthy traditional istory, and lifestyle Nutr Rev1 1997: 55111 Pt 1

UNESCO. Mediterranean diet. Inscribed in ible Cultural Heritage of Humanity. http $/ /$

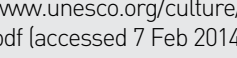

British Cardiac Society; British Hypertension 促 and cultural an udates. Pyrablic Health Nutr 2011

Chatzi L, Apostolaki G, Bibakis I, et al. Mediterranean diet on asthma and allergies among
$677-683$

D. Franks PW, Hanson RL, Knowler WC, et al. actors, and premature death. N Engl J J Me 